\title{
Editorial
}

\section{Comprehensive Management of Breast Cancer}

\section{Manzurul Haque*}

It is every woman's nightmare, a heart stopping moment, when she is declared to have been suffering from breast cancer. Her immediate concern is that she is going to die.

Breast cancer is the most common malignancy in women in Europe and North America. ${ }^{1}$ the incidence of breast cancer is rising but mortality rate has been decreasing steadily with the advent of management potential. In Bangladesh we do not know exactly, how many women are developing breast cancer or dying of it. It is not surprising that women in Bangladesh, either in villages or in cities, are the worst breast cancer sufferer owing to shyness, ignorance, social taboos and economic austerity.

Given our current understanding of breast cancer, mainly three points must be addressed adequately in regards to the management. The first step is to make an early, accurate and elaborate diagnostic work up, secondly a comprehensive and coordinated multi modality treatment protocol and thirdly prevention of breast cancer in high-risk group.

Diagnostic work up calls for a combination of careful clinical assessment and diagnostic investigations like mammography and ultrasonic scan, as well as cell cytology (FNAC) and tissue histopathology (core and open biopsy). An early diagnosis increases the possibility of cure and reduces the morbidity resulting from the disease and its treatment.
The second most important factor to be addressed is a combined, coordinated different disciplines of medical science to fight the breast cancer problem. A combined modalities approaches involving Surgery, Radiotherapy, Medical oncology, Endocrinology, Radiology, Pathology, Nursing, Social welfare and Palliative care service may actually ensure more cure and improved organ and function preservation. A multidisciplinary management is more effective and less destructive than sequential independent management of the patients.

Third component of breast cancer management is prevention of breast cancer in the high risk groups. Although a specific cause for breast cancer has not been identified, there are risk factors which increases the likelihood of development of breast cancer. The important risk factors are maternal relative with breast cancer, identification formulation over BRCA1 gene on chromosome 17 and BRCA2 gene on chromosome 13, longer reproductive span, nulliparity or late first pregnancy, previous breast cancer or endometrial cancer and obesity.

Currently the only agent to have general approval for chemoprevention of breast cancer is a selective estrogen receptor modulator (SERM) Tamoxifen ${ }^{2}$. The use of second generation of SERM Raloxifen is not recommended for prophylaxis. Other chemical agents like retinoids, aromatase inhibitors, COX-2 inhibitors lutenizing hormone releasing hormone analogues are on clinical trials and not being prescribed for prohylaxis. Surgical prophylaxis by bilateral mastectomy or bilateral 
ophorectomy are equitable options in women with very high risk of breast cancer specially with genetic mutations. ${ }^{3}$

Many of the above disciplines of management potential for breast cancer are available in our set up. To achieve the total benefit of our knowledge and skill, every woman with breast disease should have an access to the highest quality of care. With all the limitations of illiteracy, ignorance, social taboo and financial constraints it is very difficult for our women to reach the correct person at an appropriate time. For some patients, multiple visits are necessary to obtain all the required information from the various professionals involved. A multi disciplinary specialized unit may be organized at the base of medical college hospitals to provide the highest cares to the poor illiterate common people of our country suffering from breast cancers.

\section{References}

1. G. Tejler, B. Norberg, M. Dufmats, B. Nordenskjold: Survival after treatment of breast cancer in a geographically defined population. BJS 2004; 91: 1307-12.

2. Veronesi U, Maisonneuve $\mathrm{P}$, Sacchini $\mathrm{V}$, Rotmensz $\mathrm{N}$, Boyle $\mathrm{P}$. Tamoxifen for breast cancer iamong hysterectomized women. Lancet 2002; 359: 1122-1124.

3. Prichard RS, Hill ADK, Dijkstra B, McDermott EW, O'Higgins NJ. The prevention of breast cancer. BJS 2003; 90: 772-783.

All correspondence to: 